\title{
Characterization of an Acellular Scaffold for a Tissue Engineering Approach to the Nipple-Areolar Complex Reconstruction
}

\author{
Nicholas C. Pashos ${ }^{a, d}$ Michelle E. Scarritt ${ }^{a}$ Zachary R. Eagle ${ }^{a}$ \\ Jeffrey M. Gimble ${ }^{a, e}$ Abigail E. Chaffin ${ }^{b}$ Bruce A. Bunnell ${ }^{a}, c, f$ \\ ${ }^{a}$ Center for Stem Cell Research and Regenerative Medicine, Departments of b Surgery and cPharmacology, Tulane \\ University School of Medicine, 'Bioinnovation PhD Program, School of Science and Engineering, Tulane University, \\ eLaCell LLC, New Orleans, LA, and fDivision of Regenerative Medicine, Tulane National Primate Research Center, \\ Covington, LA, USA
}

\section{Keywords}

Nipple-areolar complex reconstruction - Tissue engineering •

Regenerative medicine $\cdot$ Acellular scaffold $\cdot$ Matrix

\begin{abstract}
A significant number of patients undergo mastectomies and breast reconstructions every year using many surgical-based techniques to reconstruct the nipple-areolar complex (NAC). Described herein is a tissue engineering approach that may permit a human NAC onlay graft during breast reconstruction procedures. By applying decellularization, which is the removal of cellular components from tissue, to an intact whole donor NAC, the extracellular matrix (ECM) structure of the NAC is preserved. This creates a biologically derived scaffold for cells to repopulate and regenerate the NAC. A detergent-based decellularization method was used to derive whole NAC scaffolds from nonhuman primate rhesus macaque NAC tissue. Using both histological and quantitative analyses for the native and decellularized tissues, the derived ECM graft was assessed. The bioactivity of the scaffold was evaluated following cell culture with bone marrow-derived mesenchymal stem cells (BMSCs). The data presented here demonstrate that scaffolds are devoid of cells and retain ECM integrity and a high degree of bioactivity. The con-
\end{abstract}

tent of collagen and glycosaminoglycans were not significantly altered by the decellularization process, whereas the elastin content was significantly decreased. The proliferation and apoptosis of seeded BMSCs were found to be approximately 65 and $<1.5 \%$, respectively. This study characterizes the successful decellularization of NAC tissue as compared to native NACs based on structural protein composition, lubricating protein retention, the maintenance of adhesion molecules, and bioactivity when reseeded with cells. These histological and quantitative analyses provide the foundation for a novel approach to NAC reconstruction.

(c) 2017 S. Karger AG, Basel

\section{Introduction}

Current strategies for nipple-areolar complex (NAC) reconstruction in breast cancer patients are limited to surgical techniques that create an NAC structure from existing local tissue, secondary site grafting, 3D tattooing [Halvorson et al., 2014; Jordan et al., 2014], or using com-

A portion of this paper was presented as a poster at the American Society of Gene and Cell Therapy Conference, 2015, New Orleans, LA, USA.

\section{KARGER}

(c) 2017 S. Karger AG, Basel

E-Mail karger@karger.com

www.karger.com/cto
Prof. Bruce A. Bunnell, PhD

Center for Stem Cell Research and Regenerative Medicine Department of Pharmacology, Tulane University School of Medicine 1430 Tulane Avenue, SL-99, New Orleans, LA 70112 (USA)

E-Mail bbunnell@tulane.edu 


\begin{tabular}{ll} 
Abbreviations used in this paper \\
\hline BMSCs & bone marrow-derived mesenchymal stem cells \\
ECM & extracellular matrix \\
GAGs & glycosaminoglycans \\
gDNA & genomic DNA \\
HE & hematoxylin and eosin \\
IHC & immunohistochemical \\
NAC & nipple-areolar complex \\
PCNA & proliferating cellular nuclear antigen \\
TNPRC & Tulane National Primate Research Center \\
TUNEL & TdT-mediated dUTP nick-end labeling \\
\hline
\end{tabular}

mercial acellular dermal matrix sheets [Craft and May, 2011; Seaman et al., 2012]. These surgical strategies, although offering breast reconstruction patients an NAClike structure, are highly variable. Generating a biocompatible NAC graft from decellularized human intact NAC is a promising alternative approach to reconstruction following mastectomies. Herein, a tissue-engineered, regenerative medicine approach to reconstruct the NAC using biologically derived scaffolds and autologous cell sources is described.

There are more than 2.8 million breast cancer survivors in the USA and 230,000 newly diagnosed cases per year [American Cancer Society, 2014; Howlader et al., 2014]. Approximately $36 \%$ of patients with early-stage diagnoses and $60 \%$ of patients with late-stage diagnoses undergo mastectomies [Siegel et al., 2012]. Moreover, immediate breast reconstruction following mastectomy has become more common, increasing from $20.8 \%$ in 1998 to $37.8 \%$ in 2008 [Albornoz et al., 2013]. This is not surprising, as breast reconstruction is known to provide psychological benefits for women who undergo mastectomies. There is evidence to suggest that NAC reconstruction affects psychological well-being by enhancing body image and self-esteem, or by decreasing the feeling of distress due to the mastectomy procedure itself [Al-Ghazal et al., 2000; Nano et al., 2005; Lohsiriwat and Petit, 2012]. Evidence also suggests that women are more comfortable with getting a mastectomy if the nipple can be spared or reconstructed [Didier et al., 2012]. To date, no tissue engineering strategies have been developed that focus on reconstruction of the NAC. The application of decellularization to the whole, semiglandular NAC has the potential to create a nonimmunogenic scaffold that retains the microarchitecture and gross structures of a native NAC. This tissue engineering approach to whole structure regeneration allows for the effective removal of cellular ma- terial, the preservation of the extracellular matrix (ECM) composition and structure, and retention of cell adhesion molecules [Scarritt et al., 2015]. Once decellularized, NAC scaffolds can be seeded with autologous cells to create an onlay graft that is patient specific (Fig. 1). In addition, this technique generates a whole NAC graft that aesthetically and molecularly mimics the native nipple.

\section{Materials and Methods}

\section{Decellularization}

Veterinary technicians at Tulane National Primate Research Center (TNPRC) collected rhesus macaque (Macaca mulatta) NAC tissue from disease-free animals undergoing routine euthanasia due to chronic diarrhea or self-mutilation, as well as control animals from various experiments. All animal procedures conformed to the requirements of the Animal Welfare Act and all animal protocols were approved by the Institutional Animal Care and Use Committee of the TNPRC before the implementation of experimental protocols. NAC from females and males ranging from 1-13 years of age were used.

Rhesus macaque tissue samples were decellularized through a modified protocol previously described by Bonvillain et al. [2012] and Scarritt et al. [2014]. In brief, after the NAC samples were collected, they were incubated with the Triton X-100 detergent solution and left for $2 \mathrm{~h}$ in water. Next, the NACs were incubated with the sodium deoxycholate solution (catalog No. BP349; Fisher Scientific, Fairlawn, NJ, USA) followed by a further $2 \mathrm{~h}$ in water. The samples were incubated in sodium chloride for $2 \mathrm{~h}$, followed by a 2 -h water wash. The samples were then incubated overnight at $4^{\circ} \mathrm{C}$ in a PBS solution containing $5 \times$ antibiotic/antimycotic. After overnight incubation, the samples were water washed for $2 \mathrm{~h}$, treated with DNase I (catalog No. DN25; Sigma, St. Louis, MO, USA) for $2 \mathrm{~h}$, washed with water for $2 \mathrm{~h}$, and then stored in a PBS solution containing $5 \times$ antibiotic/antimycotic at $4^{\circ} \mathrm{C}$ until use.

\section{Genomic DNA and Fragment Analysis}

The samples were frozen at $-80^{\circ} \mathrm{C}$ and then lyophilized for $48 \mathrm{~h}$ using a ModulyoD freeze dryer (Thermo Electron Corporation) set to $-40^{\circ} \mathrm{C}$ and $80 \mathrm{~mm} \mathrm{Hg}$. Using sterile tools, 3 random portions of the lyophilized samples were dissected, shredded with forceps, and weighed. The samples were processed in triplicate using a Qiagen DNeasy kit (Valencia, CA, USA) according to the manufacturer's instructions. The concentration of genomic DNA (gDNA) was quantified using a NanoDrop spectrophotometer (Thermo Fisher Scientific, Waltham, MA, USA). The gDNA recovered from all samples was precipitated by the addition of sodium acetate (final concentration $0.3 \mathrm{M}$ ) and 0.7 volumes of 2-propanol. Samples were centrifuged at $15,000 \mathrm{~g}$ at $4^{\circ} \mathrm{C}$ for $22 \mathrm{~min}$. The resulting pellet was washed with $70 \%$ ethanol, centrifuged again for $10 \mathrm{~min}$, decanted, and air dried for $15 \mathrm{~min}$. The pellet was resuspended to $1.0 \mu \mathrm{g} / \mu \mathrm{L}$ in DNA elution buffer (Qiagen DNeasy kit). gDNA fragment sizes were evaluated by gel electrophoresis through a 1.0\% UltraPure agarose gel (Invitrogen) with $0.07 \%$ ethidium bromide (Promega Corporation, Madison, WI, USA). Lastly, $1.5 \mu \mathrm{g}$ of DNA from each sample was loaded and electrophoresed at $100 \mathrm{~V}$ for $1 \mathrm{~h}$ and $15 \mathrm{~min}$. An ImageQuant LAS 4000 (GE) was used to image the gels. 


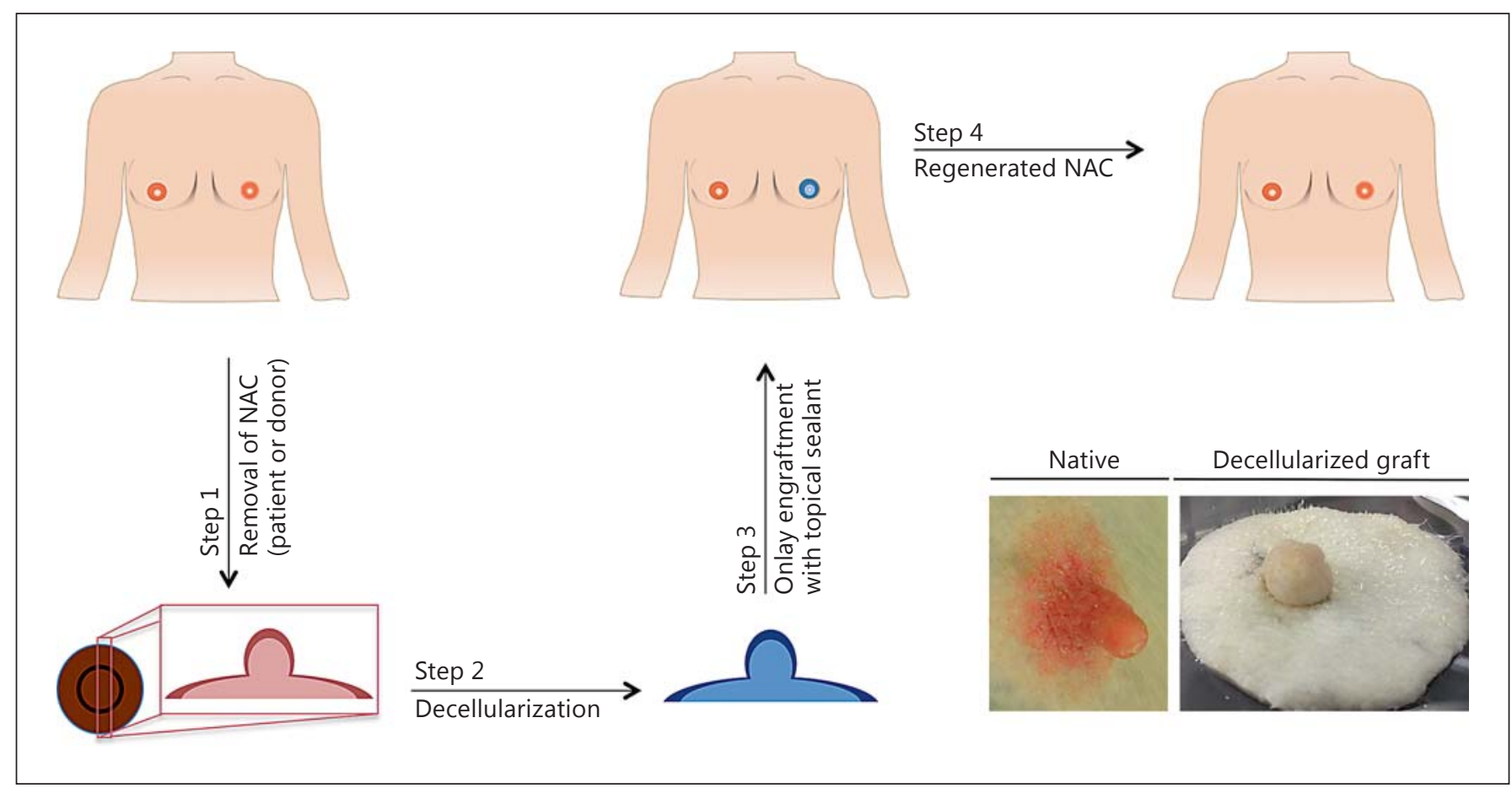

Fig. 1. Schematic of the decellularization and engraftment process of the NAC. Step 1 depicts the collection of the NAC from either the patient, who will then receive their own regenerated NAC, or a donor. Step 2 represents the decellularization of the NAC graft with detergents, salts, and enzyme. Step 3 depicts the onlay engraftment of the acellular NAC. Step 4 allows for the patient's own cells to migrate into the acellular NAC graft. Representative images of native and decellularized rhesus NAC are also provided.

\section{Histological Analysis}

Tissue embedding, sectioning, and staining were completed through the Histology Core at the Center for Stem Cell and Regenerative Medicine at Tulane University School of Medicine. Hematoxylin and eosin (HE) staining for nuclei and matrix protein structures, Gomori trichrome staining for collagen, and Movat modified pentachrome staining for elastin were accomplished using standard procedures. All histological analyses had sample sizes of 3 for each group.

Alcian blue staining for glycosaminoglycans (GAGs) was completed with GX8 (catalog No. 400460250; Acros Organics, Morris Plains, NJ, USA) and counterstained with Safranin O staining. The protocol used was a modified version of the established Abcam protocol (ab150662, Alcian blue mucin stain).

Immunohistochemical (IHC) analyses of ECM adhesion molecules employed primary murine monoclonal antibodies for laminin (catalog No. 88918; Chemicon) and fibronectin (P1h11; Iowa Hybridoma). A horseradish peroxidase conjugated goat antimouse secondary antibody (catalog No. SC-2005; Santa Cruz Biotechnology, Dallas, TX, USA) was used with all adhesion molecule IHC evaluations. Primary antibodies were used at a dilution of 1:200 and secondary antibody was used at a dilution of 1:400. An IgG1 control (catalog No. MAB398; R\&D Systems) was used to confirm the antibody specificity. After deparaffinization and rehydration through ethanol, tissue sections were boiled for $10 \mathrm{~min}$ in sodium citrate buffer. Sections were blocked for 30 min with Per-
kinElmer Blocking Reagent (catalog No. FP1136; PerkinElmer), washed 3 times with Tris-buffered saline (TBS; $50 \mathrm{mM}$ Tris, 150 $\mathrm{mM} \mathrm{NaCl}, \mathrm{pH}$ 7.6), and then incubated overnight at $4^{\circ} \mathrm{C}$ with the primary antibody or IgG1 control in a humidified chamber. The following day, samples were washed with TBS and incubated with secondary antibody at room temperature for $1 \mathrm{~h}$ in a humidified chamber. After washing with TBS, sections were counterstained with Mayer modified hematoxylin (catalog No. 1202; Newcomer Solutions, Madison, WI, USA). IHC imaging was performed using an Aperio ScanScope (Aperio, Vista, CA, USA) at a magnification of $\times 40$. The images were analyzed using the Aperio ImageScope program.

DAPI staining of tissue sections was conducted for the visualization of intact nuclei. Tissue sections were prepared as previously described. DAPI (Invitrogen) staining was imaged using a Leica DMRXA2 deconvolution inverted fluorescent microscope (Leica Microsystems, Buffalo Grove, IL, USA) fitted with the Cooke SensiCAM camera/controller and Slidebook software.

\section{Total Protein and GAG Quantification}

Samples were frozen at $-80^{\circ} \mathrm{C}$ and lyophilized. Using sterile tools, 3 random portions of the lyophilized samples were dissected, minced with forceps, and massed to approximately $100 \mathrm{mg}$ per sample. The samples were then digested and processed as previously detailed [Bonvillain et al., 2013; Scarritt et al., 2014]. 
Fig. 2. Histological stain and DAPI label of native versus decellularized NAC. Each row represents a different nuclei or ECM component stain. The first column depicts the native tissue structures and the last column depicts the decellularized matrix. HE staining: ECM is pink, nuclear material and acidic structures are purple. Movat staining: nuclei are black, elastic fibers are purple to black, collagens are yellow, mucopolysaccharides blue-green, cytoplasm and muscle are red. Gomori staining: collagen is green, nuclear and acidic structures are blue. Alcian blue staining: GAGs are light blue, nuclear material and acid structures are red. DAPI staining: nuclear material is bright blue.
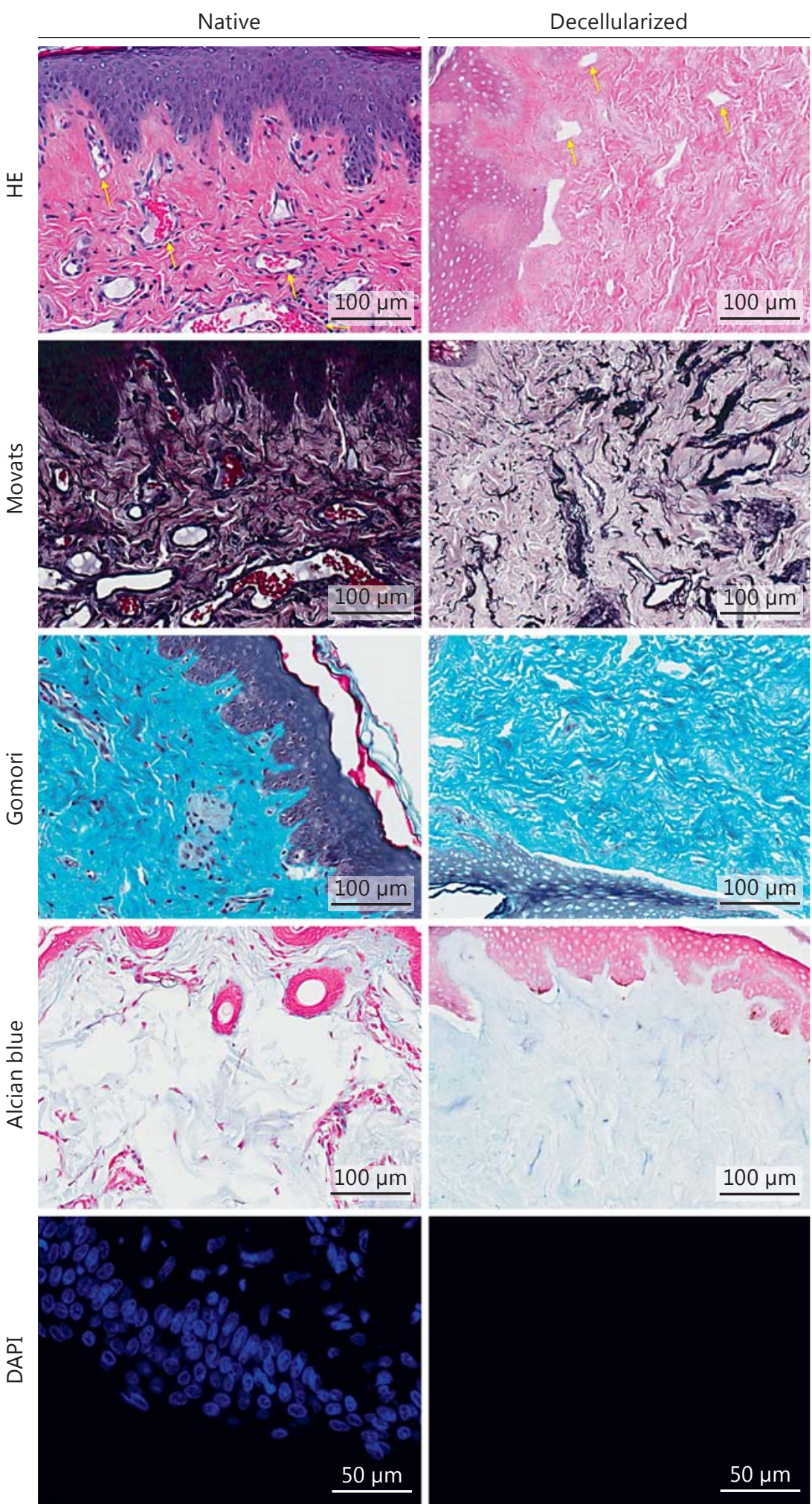


\section{Collagen Quantification}

Using the Total Collagen Kit (QuickZyme Biosciences, Leiden, The Netherlands), collagen was quantified according to the manufacturer's instructions. In brief, $10 \mathrm{mg}$ of lyophilized tissue were used for each triplicate native and acellular test group. The samples underwent hydrolysis in $6 \mathrm{M} \mathrm{HCl}$ overnight at $95^{\circ} \mathrm{C}$, followed by the oxidation of hydroxyproline. Stained hydroxyproline from samples and standards were read on a FLUOstar Optima microplate reader at $545 \mathrm{~nm}$.

\section{Elastin Quantification}

Elastin was quantified using the Fastin Elastin Assay Kit and protocol (Biocolor Life Science Assays, Carrickfergus, UK). In brief, lyophilized samples were treated with $0.25 \mathrm{M}$ of oxalic acid and incubated at $100^{\circ} \mathrm{C}$ to solubilize the elastin. Elastin was extracted from 3 incubations in oxalic acid. The extracted elastin was precipitated, concentrated, and stained. Absorbance was read at $513 \mathrm{~nm}$ using a BioRad SmartSpec Plus spectrophotometer.

\section{Dynamic Cell Seeding}

Bone marrow-derived mesenchymal stem cells (BMSCs) from rhesus macaques ( $M$. mulatta) were isolated and characterized by the TNPRC Division of the Regenerative Medicine Core. Passage 3 BMSCs were plated at 150 cells $/ \mathrm{cm}^{2}$ onto a $15-\mathrm{cm}^{2}$ tissue culturetreated dish. Acellular scaffolds were preconditioned with cell growth media in a cell culture incubator at $5 \% \mathrm{CO}_{2}$ and $95 \% \mathrm{O}_{2}$ for approximately $30 \mathrm{~min}$ prior to seeding. Three to 5 preconditioned acellular scaffolds of dimensions $7 \times 7 \times 2 \mathrm{~mm}$ were then placed into a nontreated sterile $10-\mathrm{cm}^{2}$ plate. Scaffolds were submerged in complete conditioned media ( $\alpha$-modified Eagle's medium containing $16.4 \%$ fetal bovine serum, $4 \mathrm{mM} \mathrm{L}$-glutamine, $100 \mathrm{U} / \mathrm{mL}$ penicillin, $100 \mu \mathrm{g} / \mathrm{mL}$ streptomycin, and $250 \mathrm{ng} / \mathrm{mL}$ amphotericin B). The samples were placed on an orbital rotating shaker in a tissue culture incubator and agitated at a continuous low speed. Approximately $1 \times 10^{6}$ BMSCs were then added per sample.

Seeded scaffolds were removed for analysis at 1,2, and 7 days for cell proliferation (proliferating cellular nuclear antigen; PCNA; Abcam ab29) and apoptosis (TdT-mediated dUTP nick-end labeling; TUNEL). The TUNEL protocol was performed as previously described [Bonvillain et al., 2012]. For assessing cell proliferation, tissue sections were blocked for 15 min with 5\% BSA, incubated with primary antibody at a dilution of 1:6,000, washed with TBS, and then incubated with secondary antibody at a concentration of 1:200 (Alexa Fluor 488, goat anti-mouse; Santa Cruz). Cell proliferation $(n=4)$ and apoptosis $(n=4)$ were imaged using a Leica DMRXA2 deconvolution inverted fluorescent microscope (Leica Microsystems, Buffalo Grove, IL, USA) fitted with the Cooke SensiCAM camera/controller and Slidebook software. Ten images at $\times 10$ magnification were taken of each biological replicate and blind counted.

Live/dead assay of the NAC scaffold was performed in 6-well plates with a cellular density of approximately $3.5 \times 10^{5}$ per well, with a 5 -mm-dimeter and 2- to 3 -mm-deep scaffold in each well. NAC scaffolds were taken from 3 donor nonhuman primates $(n=$ $4)$, a no scaffold control $(n=4)$, and a commercially available porcine dermal scaffold, Strattice $(n=4)$. Using trypan blue exclusion, cell viability was evaluated at 12, 24 and $48 \mathrm{~h}$ after cell adhesion. All media were collected, wells were trypsinized for 3 min, neutralized with an equal volume of cell culture media and combined with previously collected media. Cells were stained using trypan blue

Tissue Engineering Approach to the

Nipple-Areolar Complex Reconstruction
(0.4\%; Gibco 15250) and counted on a hemocytometer. Cell viability $([1-($ dead cell count/total cell count $)] \times 100 \%)$ was calculated per well and averaged for each group.

\section{Statistics}

Statistical significance was determined by 2-tailed $t$ tests or 1-way ANOVA with Tukey's Post-hoc. Three or more experiments were conducted for all analyses. A $p$ value $\leq 0.05$ was considered significant. Numerical values are presented as the mean \pm SEM. All calculations and graphs were completed in GraphPad Prism version 5.03.

\section{Results}

\section{Nuclei Are Removed by Decellularization}

$\mathrm{HE}$ staining of native NAC tissue showed dense eosin staining in the keratinocyte layer of the epidermis (Fig. 2). Blood vessel structures, surrounded by darkly stained cell nuclei in the native samples, are still visible in the decellularized NAC (Fig. 2, yellow arrows). Decellularized macaque NAC tissue showed a lack of HE-stained nuclei indicating efficient cell removal (Fig. 2). Complete cell removal was also observed within areas of dense collagen (Fig. 2). DAPI staining of decellularized tissue sections revealed that there were no intact nuclei in NAC scaffolds as compared to the native NAC, which showed heavy nuclear staining throughout the tissue (Fig. 2).

\section{gDNA Isolation, Quantification, and Fragment Analysis Reveals Decellularization Reduces DNA Content}

Native NAC contained 1,905 $\pm 422.1 \mathrm{ng}$ DNA/mg lyophilized tissue, while decellularized NAC contained significantly less DNA at $56.51 \pm 8.45 \mathrm{ng} / \mathrm{mg}$. Gel electrophoresis of $1 \mu \mathrm{g}$ of pooled gDNA from native NAC appeared as a dark, dense, high-molecular-weight band, whereas pooled DNA isolated from decellularized NAC did not create any visible banding, consistent with degradation and/or removal (Fig. 3).

\section{ECM Structure and Composition following Decellularization}

NAC tissue sections were stained with Gomori trichrome for collagen, Movat modified pentachrome for elastin, and Alcian blue for GAGs. Collagen fibers (green) were well preserved throughout decellularized NAC in a pattern comparable to native NAC (Fig. 2). Similarly, elastin and GAGs were also visible in decellularized NAC; however, the staining for elastin and GAGs was less intense in decellularized NAC in comparison to native 


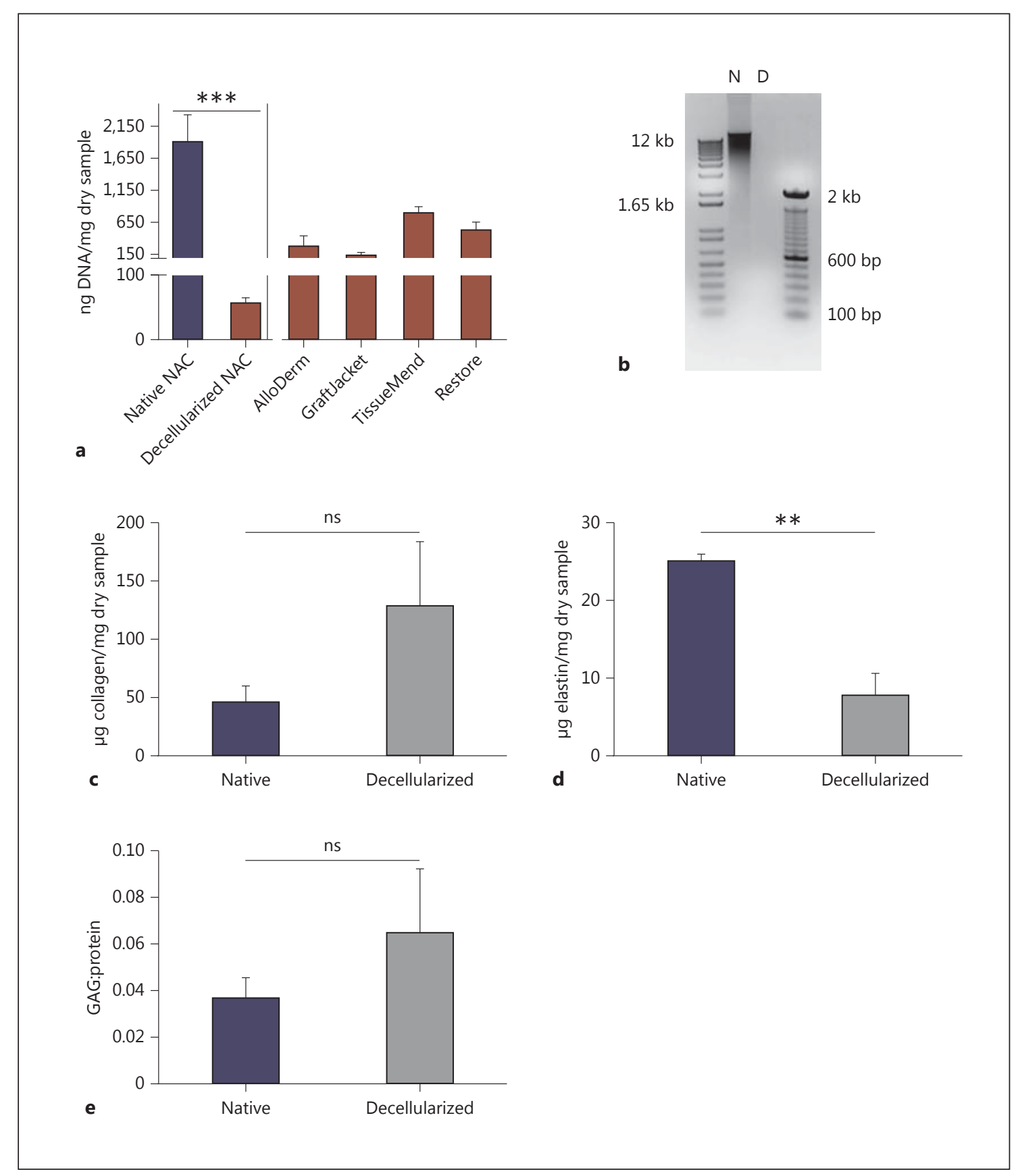

Fig. 3. a DNA quantification: average values of lyophilized tissue of native and decellularized matrix $(1,905 \pm 422.1$ and $56.51 \pm 8.45$ ng DNA/mg, respectively), with a significant difference of $* * * p \leq$ 0.001 . The commercially available acellular products TissueMend $^{\mathrm{TM}}$, Restore ${ }^{\mathrm{TM}}$, GraftJacket ${ }^{\mathrm{TM}}$, and AlloDerm ${ }^{\mathrm{TM}}$ have reported DNA contents of 794.6 $\pm 97.8,526.8 \pm 125.6,134.6 \pm 44.0$, and 273 $\pm 169 \mathrm{ng}$ DNA/mg dry tissue weight, respectively [Choe and Bell, 2001; Derwin et al., 2006; Moore et al., 2015]. b DNA fragment length: agarose gel of native (N) and decellularized (D) nipple scaffold. c Collagen quantification: average collagen values of $46.12 \pm$ 13.88 and $128.57 \pm 55.05 \mu \mathrm{g}$ collagen/mg dry sample for native and decellularized NAC, respectively. $\mathbf{d}$ Elastin quantification values of $7.77 \pm 2.84$ and $25.03 \pm 0.93 \mu \mathrm{g} / \mathrm{mg}$ dry tissue for native and decellularized NAC, respectively. ${ }^{* *} p \leq 0.01$. e GAGs to total protein concentrations, with no significant difference between native and decellularized NAC $(0.068 \pm 0.027$ and $0.025 \pm 0.004$, respectively). 


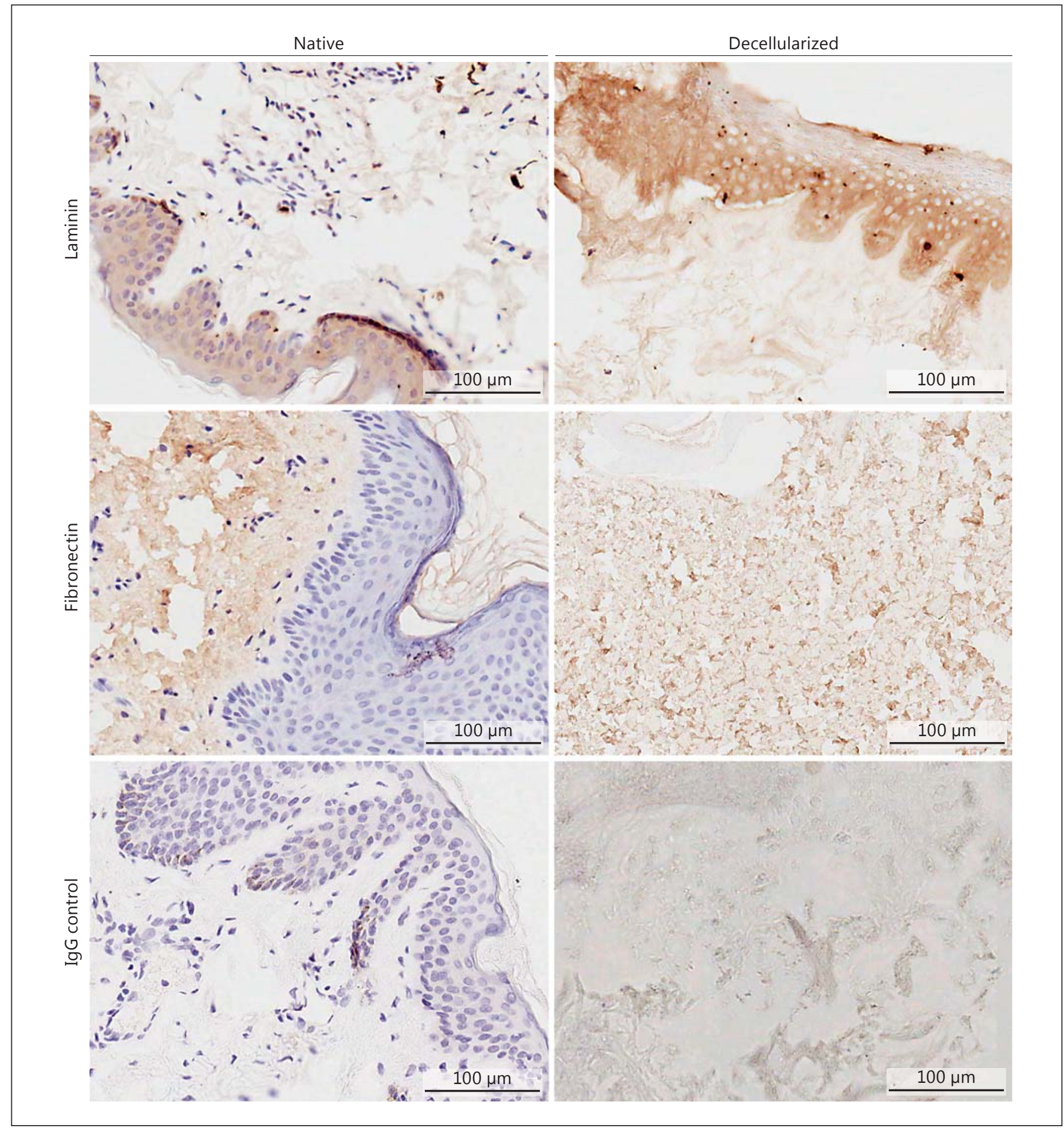

Fig. 4. Immunohistologic labeling of cell adhesion molecules of native versus decellularized NAC. Each row represents a different nuclei or ECM component stain. The first column depicts the native tissue structures and the second column depicts the decellular- ized matrix. The secondary antibody with an HRP attached was developed with $\mathrm{DAB}$, which developed the antigen of interest in a brown color. The counterstain of the nuclei is shown as purple staining. 


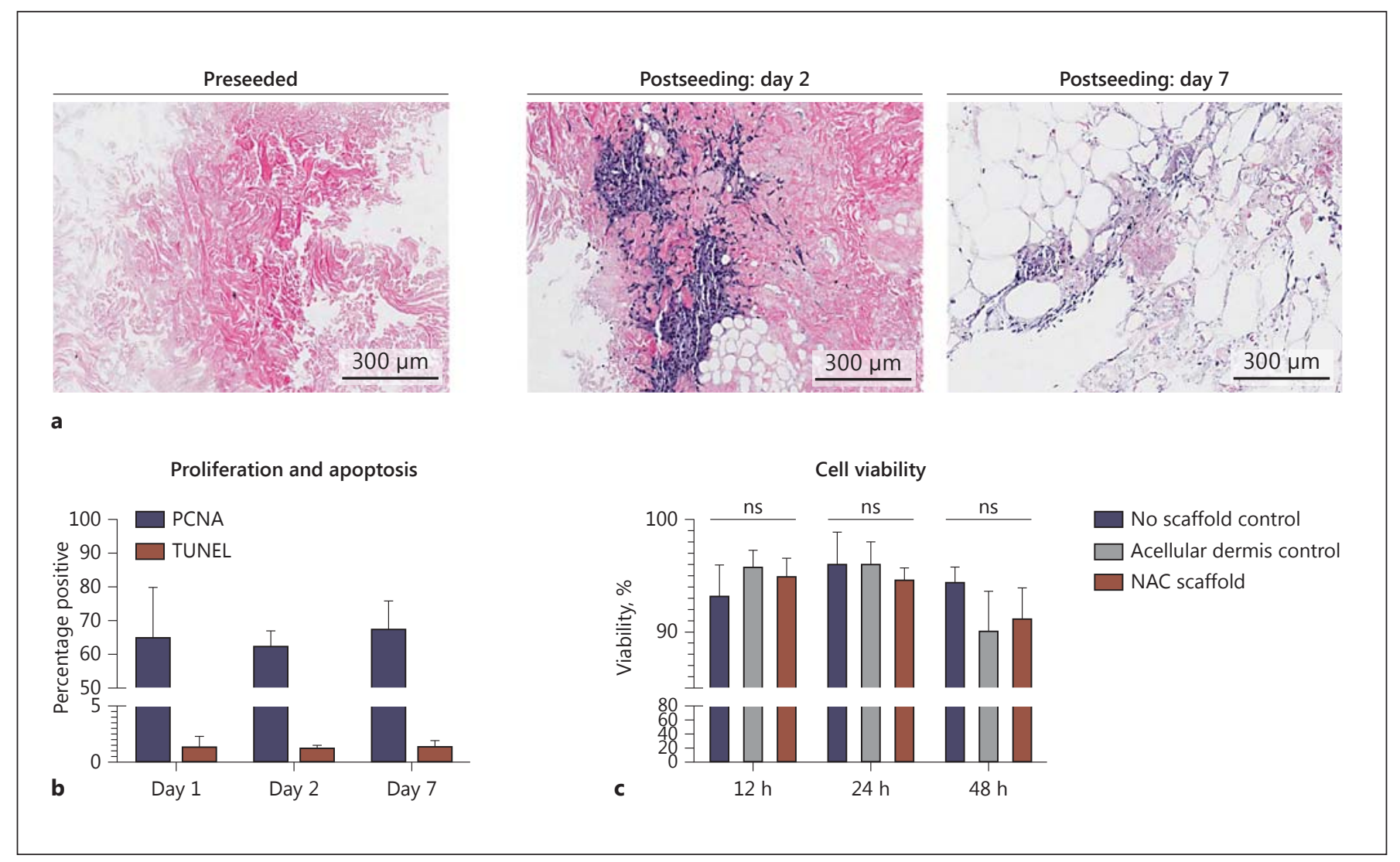

Fig. 5. Cell proliferation and apoptosis, and cell viability. a The acellular NAC scaffolds initially added to culture, and the migration of the cells into areas of the NAC scaffold on days 2 and 7. b PCNA $(64.98 \pm 7.42,62.38 \pm 2.34$, and $67.42 \pm 4.23 \%$, respectively) and TUNEL $(1.34 \pm 0.48,1.21 \pm 0.14$, and $1.34 \pm 0.3 \%$, respectively) on days 1,2 , and 7 postseeding of cells on the acellu- lar NAC. c Cell viability at 12, 24, and 48 h [no scaffold control: $93.18 \pm 1.39 \%(12 \mathrm{~h}), 95.98 \pm 1.45 \%(24 \mathrm{~h})$, and $94.39 \pm 0.69 \%$ $(48 \mathrm{~h})$; commercially available acellular dermis control: $95.18 \pm$ $0.79 \%(12 \mathrm{~h}), 95.97 \pm 1.03 \%(24 \mathrm{~h})$, and $90.39 \pm 1.18 \%(48 \mathrm{~h})$; decellularized NAC scaffold: $94.92 \pm 0.95 \%$ (12 h), $94.59 \pm 0.65 \%$ (24 h), and $91.14 \pm 1.62 \%(48 \mathrm{~h})]$.
NAC. This decrease may be attributed, in part, to the removal of cellular content. To evaluate whether decellularization removes or damages collagen, elastin, or GAGs, colorimetric quantification assays were executed.

Native and decellularized NAC contained $46.12 \pm$ 13.88 and $128.57 \pm 55.05 \mu \mathrm{g}$ collagen/mg dry tissue, respectively, indicating that the collagen content was enriched, but not significantly (Fig. 2, 3). Soluble tropoelastins, lathyrogenic elastin, $\alpha$-elastin, and $\mathrm{k}$-elastin in native and decellularized NAC was quantified using a colorimetric assay. Decellularization significantly reduced the elastin content from $25.03 \pm 0.93$ to $7.77 \pm 2.84 \mu \mathrm{g} / \mathrm{mg}$ of dry tissue (Fig. 3). GAGs in native and decellularized NAC tissues were quantified using a colorimetric assay. The GAG content was measured as a ratio to total protein concentrations and was determined to be $0.037 \pm 0.009$ for the native tissue and $0.065 \pm 0.027$ for the decellularized tissue (Fig. 3). There was no significant decrease in GAG content after decellularization.

To determine the presence and localization of the cell adhesion molecules fibronectin and laminin, immunohistochemistry was used for the visual analysis. Laminin was found to be present throughout the matrix of both the native and decellularized NAC with concentrated staining in the epidermis where a dense layer of keratinocytes resides (Fig. 4). Fibronectin was also seen ubiquitously throughout the acellular and native samples, but was most concentrated in the dermis layers (Fig. 4). Both secondary antibody only and serotype (IgG1) controls confirmed that there was minimal background staining and nonspecific staining present (Fig. 4). 
Dynamic Cell Seeding of Stem Cells Demonstrates the Bioactivity of Decellularized NAC

HE staining of reseeded NAC tissue sections showed that cells initially attached to the periphery of the scaffold at days 1 and 2. As culture progressed, cells migrated deeper into areas of the NAC scaffold by day 7 (Fig. 5). Seeded cells were evaluated for both cell proliferation and apoptosis after 1, 2, and 7 days of dynamic culture on NAC scaffolds. Less than $1 \%$ of the cells underwent apoptosis during the culture period. The percentage of cells undergoing proliferation was found to be $64.98 \pm 7.42$, $62.38 \pm 2.34$, and $67.42 \pm 4.23 \%$ for day 1,2 , and 7 , respectively (Fig. 5). Additionally, cell viability was found to be greater than $90 \%$ for the 12,24 , and 48 -h time points for all groups [no scaffold control: $93.18 \pm 1.39 \%$ (12 h), 95.98 $\pm 1.45 \%(24 \mathrm{~h}), 94.39 \pm 0.69 \%(48 \mathrm{~h})$; commercially available acellular dermis control: $95.18 \pm 0.79 \%(12 \mathrm{~h}), 95.97$ $\pm 1.03 \%$ ( $24 \mathrm{~h}$ ), $90.39 \pm 1.18 \%$ ( $48 \mathrm{~h}$ ); decellularized NAC scaffold: $94.92 \pm 0.95 \%$ (12 h), $94.59 \pm 0.65 \%$ ( $24 \mathrm{~h}$ ), 91.14 $\pm 1.62 \%(48 \mathrm{~h})]$.

\section{Discussion}

The lack of tissue engineering approaches for breast reconstruction led us to explore the potential application of decellularization to the structural and physical aesthetic uniqueness of the NAC. To achieve the overall goal of applying a decellularized whole NAC during the reconstruction process, the efficacy of the decellularization process was evaluated with respect to the removal of intact cells and debris without damaging the ECM. We have previously shown that nonhuman primate and rat lung tissues can be efficiently decellularized using a combination of detergents, salts, and enzymes [Bonvillain et al., 2012, 2013; Scarritt et al., 2014]; however, decellularization of NAC posed distinct challenges, which included the lack of an accessible vasculature for the distribution of decellularization solutions throughout the tissue. To address this challenge, the incubation time, detergent concentration, and physical agitation were optimized to permit the efficient decellularization of NAC. The efficacy of NAC decellularization was evaluated based on 3 key criteria, as outlined by Crapo et al. [2011]: (1) no visible signs of cells or cell debris, (2) a significant reduction of DNA, and (3) degradation of any remaining DNA to less than $200 \mathrm{bp}$. These criteria are based on the principle that cellular debris, substantially high concentrations of DNA, or the presence of long DNA fragments in a decellularized tissue are potentially immunogenic and, there-

Tissue Engineering Approach to the Nipple-Areolar Complex Reconstruction fore, possibly detrimental to the successful incorporation posttransplantation. Decellularized human dermal products have been used clinically for over 30 years with no substantial evidence of immune rejection by the host [Wainwright et al., 1996; Harirchian and Baredes, 2013; Scarritt et al., 2015]. The lack of immunogenicity of acellular dermal matrices has permitted them to be used in an estimated $56 \%$ of prosthetic breast reconstructions [Ho et al., 2012; Janis et al., 2012].

Decellularized rhesus macaque NAC tissue sections stained with HE and DAPI showed no intact NAC nuclei. A greater than $97 \%$ reduction in gDNA was observed as compared to the native control, which is a significant minimization of the DNA content to less $56 \mathrm{ng} \mathrm{DNA} / \mathrm{mg}$ of dried tissue weight. Currently there are commercially available acellular products, such as TissueMend ${ }^{\mathrm{TM}}$, Restore $^{\mathrm{TM}}$, GraftJacket ${ }^{\mathrm{TM}}$, and AlloDerm ${ }^{\mathrm{TM}}$, which have reported DNA contents of $794.6 \pm 97.8,526.8 \pm 125.6,134.6$ \pm 44.0 , and $273 \pm 169 \mathrm{ng} \mathrm{DNA} / \mathrm{mg}$ dry tissue weight, respectively [Choe and Bell, 2001; Derwin et al., 2006; Moore et al., 2015]. Our observed residual DNA content in the NAC acellular graft is well below the threshold that is currently offered commercially and accepted clinically.

By maintaining both the micro and macro structures of the native tissue, the decellularized whole matrix offers a major potential advantage to the reconstruction of the NAC by allowing for an engineered tissue that aesthetically mimics that of the patients' preoperative anatomy. To achieve this goal, it is essential that the extracellular components, such as collagen, elastin, GAGs, and cell adhesion molecules, are minimally disturbed during the decellularization process. Histological analysis through HE showed that the primary structures of the NAC are maintained on the microscopic scale; the ECM fibers were intact and no nuclei were found in the acellular NAC. Gomori trichrome staining showed that collagen, a major ECM component for the skin, was preserved. This was confirmed by colorimetric quantification of collagen hydroxyproline. From Movat pentachrome staining, elastin fibers were visible in the decellularized NAC; however, the overall staining intensity, in comparison to native $\mathrm{NAC}$, indicated a reduction in elastin. Colorimetric quantification confirmed that decellularization reduced the soluble elastin content of the NAC. Although this reduction in elastin may affect the mechanical parameters of the NAC and will be evaluated in future studies, this was an expected outcome of the detergent-based decellularization process. Elastin has been shown in previous studies to be significantly decreased during decellularization in a variety of tissue types [Petersen et al., 2012; Reimer et 
al., 2016]. However, it has also been observed that the native environment in vivo can restore levels of elastin and other extracellular proteins, such as collagens, after scaffolds are reincorporated into the body [Ghazanfari et al., 2015; Reimer et al., 2016]. Alcian blue staining of decellularized NAC samples confirmed the presence of GAGs, which are an important ECM component for lubrication. A slight decrease in the Alcian blue staining intensity suggested that some GAG content may have been lost during the decellularization process. However, GAG quantification using a colorimetric assay showed no significant decrease. Since proteoglycans are localized in both cells and ECM, this leads to an expectation that the GAGs would have some decrease after decellularization [Ferdous and Grande-Allen, 2007; Petersen et al., 2012]. These trends with respect to the color intensity for Gomori, Movat, GAGs and/or the ratio of GAG to total protein concentration were similar to those observed in rhesus macaque lung, rat lung, and porcine dermis [Hoganson et al., 2010; Bonvillain et al., 2012; Scarritt et al., 2014]. Overall, these histologic analyses confirmed that the resulting NAC acellular matrix maintained its structure, shape, and composition.

The presence of cellular adhesion molecules in the decellularized matrix provides a basis for cellular attachment to and cellular migration in the acellular scaffold, enhancing its bioactivity. In accordance with Hoganson et al. [2010] and Walter et al. [1998], who previously demonstrated the preservation of fibronectin and laminin after decellularization of the porcine dermis, we observed that both laminin and fibronectin were preserved; however, they were differentially localized. Laminin was observed predominantly in the epidermis while fibronectin was expressed ubiquitously throughout the dermis.

To determine the bioactivity of the acellular NAC, the scaffold was seeded with rhesus macaque BMSCs. Cells were able to survive and proliferate throughout the 7 -day culture with low percentages of cell death. Similarly, cell viability was found to be greater than $90 \%$ and no significant difference was observed between the NAC scaffold, the commercially available acellular dermis, and the no scaffold control. The HE-stained tissue sections show BMSCs attached to the periphery of the scaffold during early time points with cells migrating deeper into areas of the acellular scaffold by day 2 and 7 . This preliminary evaluation of cell seeding indicated that the decellularized scaffold provides an environment permissible for the adhesion and continuous growth of the seeded cells.

\section{Conclusions}

Whole NAC reconstruction using a tissue engineered acellular graft may provide a structural and cosmetic recreation of a patient's own NAC, thereby surpassing the currently available standard of care. The current study serves as an initial in vitro report establishing the feasibility of NAC tissue engineering strategies. Herein, the characterization of the decellularized NAC scaffold with comparison to the native NAC structure is reported as well as analyses of the isolated NAC scaffold's cellular bioactivity. This preliminary study sets the stage for further biological and material characterization of the acellular NAC graft, as well as the optimization of the decellularization process in anticipation of future clinical translation. Future in vivo studies will focus on the rate of NAC engraftment and neovascularization, using skin wound healing models with commercially available dermal matrices as a standard of care control comparison.

\section{Acknowledgements}

Nicholas C. Pashos is supported by an NSF IGERT Bioinnovation training grant (DGE-1144646). These studies were partially supported by the following grant NIH/OD P51OD011104. The authors would like to thank Dina Gaupp, Maurice Duplantis, and Cyndi Trygg for their technical assistance.

\section{Author Contributions}

N.C.P., M.E.S., B.A.B., J.M.G., and A.C. helped to initially design, write and/or edit the report. N.C.P., M.E.S., and Z.R.E. carried out the experiments.

\section{Disclosure Statement}

B.A. Bunnell and N.C. Pashos are the listed inventors of a patent application filed with assignee of Tulane University, based on the data presented in this report. J.M. Gimble is the cofounder, coowner, and chief scientific officer of LaCell LLC.

\section{References}

Al-Ghazal, S.K., L. Fallowfield, R.W. Blamey (2000) Comparison of psychological aspects and patient satisfaction following breast conserving surgery, simple mastectomy and breast reconstruction. Eur J Cancer 36: 19381943.

Albornoz, C.R., P.B. Bach, B.J. Mehrara, J.J. Disa, A.L. Pusic, C.M. McCarthy, P.G. Cordeiro, E. Matros (2013) A paradigm shift in U.S. breast reconstruction: increasing implant rates. Plast Reconstr Surg 131: 15-23.

Pashos/Scarritt/Eagle/Gimble/Chaffin/ Bunnell 
American Cancer Society (2014) Cancer Facts \& Figures 2014. Atlanta, American Cancer Society.

Bonvillain, R.W., S. Danchuk, D.E. Sullivan, A.M. Betancourt, J.A. Semon, M.E. Eagle, J.P. Mayeux, A.N. Gregory, G. Wang, I.K. Townley, Z.D. Borg, D.J. Weiss, B.A. Bunnell (2012) A nonhuman primate model of lung regeneration: detergent-mediated decellularization and initial in vitro recellularization with mesenchymal stem cells. Tissue Eng Part A 18: 2437-2452.

Bonvillain, R.W., M.E. Scarritt, N.C. Pashos, J.P. Mayeux, C.L. Meshberger, A.M. Betancourt, D.E. Sullivan, B.A. Bunnell (2013) Nonhuman primate lung decellularization and recellularization using a specialized large-organ bioreactor. J Vis Exp 82: e50825.

Choe, J.M., T. Bell (2001) Genetic material is present in cadaveric dermis and cadaveric fascia lata. J Urol 166: 122-124.

Craft, R.O., J.W. May (2011) Staged nipple reconstruction with vascularized SurgiMend acellular dermal matrix. Plast Reconstr Surg 127: $148 \mathrm{e}-149 \mathrm{e}$.

Crapo, P.M., T.W. Gilbert, S.F. Badylak (2011) An overview of tissue and whole organ decellularization processes. Biomaterials 32: 32333243.

Derwin, K.A., A.R. Baker, R.K. Spragg, D.R. Leigh, J.P. Iannotti (2006) Commercial extracellular matrix scaffolds for rotator cuff tendon repair: biomechanical, biochemical, and cellular properties. J Bone Joint Surg Am 88: 2665-2672.

Didier, F., P. Arnaboldi, S. Gandini, A. Maldifassi, A. Goldhirsch, D. Radice, I. Minotti, B. Ballardini, A. Luini, B. Santillo, M. Rietjens, J.Y. Petit (2012) Why do women accept to undergo a nipple sparing mastectomy or to reconstruct the nipple areola complex when nipple sparing mastectomy is not possible? Breast Cancer Res Treat 132: 1177-1184.

Ferdous, Z., K.J. Grande-Allen (2007) Utility and control of proteoglycans in tissue engineering. Tissue Eng 13: 1893-1904.
Ghazanfari, S., A. Driessen-Mol, B. Sanders, P.E. Dijkman, S.P. Hoerstrup, F.P. Baaijens, C.V. Bouten (2015) In vivo collagen remodeling in the vascular wall of decellularized stented tissue-engineered heart valves. Tissue Eng Part A 21: 2206-2215.

Halvorson, E.G., M. Cormican, M.E. West, V. Myers (2014) Three-dimensional nipple-areola tattooing: a new technique with superior results. Plast Reconstr Surg 133: 1073-1075.

Harirchian, S., S. Baredes (2013) Use of AlloDerm in primary reconstruction after resection of squamous cell carcinoma of the lip and oral commissure. Am J Otolaryngol 34: 611-613.

Ho, G., T.J. Nguyen, A. Shahabi, B.H. Hwang, L.S Chan, A.K. Wong (2012) A systematic review and meta-analysis of complications associated with acellular dermal matrix-assisted breast reconstruction. Ann Plast Surg 68: 346-356.

Hoganson, D.M., E.M. O’Doherty, G.E. Owens, D.O. Harilal, S.M. Goldman, C.M. Bowley, C.M. Neville, R.T. Kronengold, J.P. Vacanti (2010) The retention of extracellular matrix proteins and angiogenic and mitogenic cytokines in a decellularized porcine dermis. Biomaterials 31: 6730-6737.

Howlader, N., A.M. Noone, M. Krapcho, J. Garshell, D. Miller, S.F. Altekruse, C.L. Kosary, M. Yu, J. Ruhl, Z. Tatalovich, A. Mariotto, D.R. Lewis, H.S. Chen, E.J. Feuer, K.A. Cronin (eds) (2014) SEER Cancer Statistics Review, 1975-2011. Bethesda, National Cancer Institute.

Janis, J.E., A.C. O'Neill, J. Ahmad, T. Zhong, S.O. Hofer (2012) Acellular dermal matrices in abdominal wall reconstruction: a systematic review of the current evidence. Plast Reconstr Surg 130(5 suppl 2): 183S-193S.

Jordan, S.W., N. Khavanin, N.A. Fine, J.Y. Kim (2014) An algorithmic approach for selective acellular dermal matrix use in immediate two-stage breast reconstruction: indications and outcomes. Plast Reconstr Surg 134: 178188.

Lohsiriwat, V., J. Petit (2012) Nipple sparing mastectomy: from prophylactic to therapeutic standard. Gland Surg 1: 75-79.

Moore, M.A., B. Samsell, G. Wallis, S. Triplett, S. Chen, A.L. Jones, X. Qin (2015) Decellularization of human dermis using non-denaturing anionic detergent and endonuclease: a review. Cell Tissue Bank 16: 249-259.
Nano, M.T., P.G. Gill, J. Kollias, M.A. Bochner, P Malycha, H.R. Winefield (2005) Psychological impact and cosmetic outcome of surgical breast cancer strategies. ANZ J Surg 75: $940-$ 947

Petersen, T.H., E.A. Calle, M.B. Colehour, L.E. Niklason (2012) Matrix composition and mechanics of decellularized lung scaffolds. Cells Tissues Organs 195: 222-231.

Reimer, J., Z. Syedain, B. Haynie, M. Lahti, J. Berry, R. Tranquillo (2016) Implantation of a tissue-engineered tubular heart valve in growing lambs. Ann Biomed Eng, Epub ahead of print.

Scarritt, M.E., R.W. Bonvillain, B.J. Burkett, G. Wang, E.Y. Glotser, Q. Zhang, M.C. Sammarco, A.M. Betancourt, D.E. Sullivan, B.A. Bunnell (2014) Hypertensive rat lungs retain hallmarks of vascular disease upon decellularization but support the growth of mesenchymal stem cells. Tissue Eng Part A 20: 1426-1443.

Scarritt, M.E., N.C. Pashos, B.A. Bunnell (2015) A review of cellularization strategies for tissue engineering of whole organs. Front Bioeng Biotechnol 3: 43.

Seaman, B.J., S.R. Akbari, S.P. Davison (2012) A novel technique for nipple-areola complex reconstruction: the acellular dermal matrix onlay graft. Plast Reconstr Surg 129: 580e-581e.

Siegel, R., C. DeSantis, K. Virgo, K. Stein, A. Mariotto, T. Smith, D. Cooper, T. Gansler, C. Lerro, S. Fedewa, C. Lin, C. Leach, R.S. Cannady, H. Cho, S. Scoppa, M. Hachey, R. Kirch, A. Jemal, E. Ward (2012) Cancer treatment and survivorship statistics, 2012. CA Cancer J Clin 62: 220-241.

Wainwright, D., M. Madden, A. Luterman, J. Hunt, W. Monafo, D. Heimbach, R. Kagan, K. Sittig, A. Dimick, D. Herndon (1996) Clinical evaluation of an acellular allograft dermal matrix in full-thickness burns. J Burn Care Rehabil 17: 124-136.

Walter, R.J., T. Matsuda, H.M. Reyes, J.M. Walter, M. Hanumadass (1998) Characterization of acellular dermal matrices (ADMs) prepared by two different methods. Burns 24: 104-113.
Tissue Engineering Approach to the Nipple-Areolar Complex Reconstruction
Cells Tissues Organs 2017;203:183-193 DOI: $10.1159 / 000455070$ 